\title{
CONFORMALITY AND ISOMETRY OF RIEMANNIAN MANIFOLDS TO SPHERES $\left({ }^{1}\right)$
}

\author{
BY \\ CHUAN-CHIH HSIUNG $\left({ }^{2}\right)$ AND LOUIS W. STERN
}

\begin{abstract}
Suppose that a compact Riemannian manifold $M^{n}$ of dimension $n>2$ admits an infinitesimal nonisometric conformal transformation $v$. Some curvature conditions are given for $M^{n}$ to be conformal or isometric to an $n$-sphere under the initial assumption that $L_{v} R=0$, where $L_{v}$ is the operator of the infinitesimal transformation $v$ and $R$ is the scalar curvature of $M^{n}$. For some special cases, these conditions were given by Yano [10] and Hsiung [2].
\end{abstract}

1. Introduction. Let $M^{n}$ be a Riemannian manifold of dimension $n \geqq 2$ and class $C^{3},\left(g_{i j}\right)$ the symmetric matrix of the positive definite metric of $M^{n}$, and $\left(g^{i j}\right)$ the inverse matrix of $\left(g_{i j}\right)$, and denote by $\nabla_{i}, R_{h i j k}, R_{i j}=R^{k}{ }_{i j k}$ and $R=g^{i j} R_{i j}$ the operator of covariant differentiation with respect to $g_{i j}$, the Riemann tensor, the Ricci tensor and the scalar curvature of $M^{n}$ respectively. Let $d$ be the operator of exterior derivation, $\delta$ the operator of coderivation, and $\Delta=d \delta+\delta d$ the LaplaceBeltrami operator. Throughout this paper all Latin indices take the values $1, \ldots, n$ unless stated otherwise. We shall follow the usual tensor convention that indices can be raised and lowered by using $g^{i j}$ and $g_{i j}$ respectively, and that repeated indices imply summation.

Let $v$ be a vector field defining an infinitesimal conformal transformation on $M^{n}$. Denote by the same symbol $v$ the 1 -form corresponding to the vector field $v$ by the duality defined by the metric of $M^{n}$, and by $L_{v}$ the operator of the infinitesimal transformation $v$. Then we have

$$
L_{v} g_{i j}=\nabla_{i} v_{j}+\nabla_{j} v_{i}=2 \rho g_{i j} .
$$

The infinitesimal transformation $v$ is said to be homothetic or an infinitesimal isometry according as the scalar function $\rho$ is constant or zero. On a compact orientable Riemannian manifold, an infinitesimal homothetic transformation is necessarily an infinitesimal isometry; see [9]. We also denote by $L_{d \rho}$ the operator of the infinitesimal transformation generated by the vector field $\rho^{i}$ defined by

$$
\rho^{i}=g^{i j} \rho_{j}, \quad \rho_{j}=\nabla_{j} \rho .
$$

Received by the editors June 29, 1970.

AMS 1970 subject classifications. Primary 53A30, 53C20; Secondary 54H15.

Key words and phrases. Infinitesimal nonisometric conformal transformations, scalar curvature, lengths of Riemann and Ricci curvature tensors.

( $\left.{ }^{1}\right)$ The contents of this paper were published as a Research Announcement in Bull. Amer. Math. Soc. 76 (1970), 1253-1256.

${ }^{(2)}$ The work of this author was partially supported by NSF grant GP-11965.

Copyright (C) 1972, American Mathematical Society 
Let $\xi_{I(p)}$ and $\eta_{I(p)}$ be two tensor fields of the same order $p \leqq n$ on a compact orientable manifold $M^{n}$, where $I(p)$ denotes an ordered subset $\left\{i_{1}, \ldots, i_{p}\right\}$ of the set $\{1, \ldots, n\}$ of positive integers less than or equal to $n$. Then the local and global scalar products $\langle\xi, \eta\rangle$ and $(\xi, \eta)$ of the tensor fields $\xi$ and $\eta$ are defined by

$$
\begin{aligned}
& \langle\xi, \eta\rangle=(1 / p !) \xi^{I(p)} \eta_{I(p)}, \\
& (\xi, \eta)=\int_{M^{n}}\langle\xi, \eta\rangle d V,
\end{aligned}
$$

where $d V$ is the element of volume of the manifold $M^{n}$ at a point. We also define

$$
\|\xi\|=p !\langle\xi, \xi\rangle \text {. }
$$

From (1.3) and (1.4) it follows that $(\xi, \xi)$ is nonnegative, and that $(\xi, \xi)=0$ implies that $\xi=0$ on the whole manifold $M^{n}$.

In the last decade or so various authors have studied the conditions for a Riemannian manifold $M^{n}$ of dimension $n>2$ with constant scalar curvature $R$ to be either conformal or isometric to an $n$-sphere. Very recently Yano, Obata, Hsiung and Mugridge (see [13], [10], [4]) have been able to extend some of these results by replacing the constancy of $R$ by $L_{u} R=0$, where $u$ is a certain vector field on $M^{n}$. The purpose of this paper is to continue their work, in particular Yano's [10], by establishing the following theorems.

To begin we denote by $(C)$ the following condition:

(C) A compact Riemannian manifold $M^{n}$ of dimension $n>2$ admits an infinitesimal nonisometric conformal transformation $v$ satisfying (1.1) with $\rho \neq 0$ such that $L_{v} R=0$.

THEOREM I. An orientable $M^{n}$ is conformal to an $n$-sphere if it satisfies condition (C) and

$$
\begin{aligned}
& \left(\rho_{i} \rho^{i}-\frac{1}{n-1} R \rho^{2}, R\right) \geqq 0, \\
& L_{v}\left(a^{2} A+\frac{c-4 a^{2}}{n-2} B\right)=0,
\end{aligned}
$$

where $A$ and $B$ are defined by

$$
A=R^{h i j k} R_{h i j k}, \quad B=R^{i j} R_{i j},
$$

and $a, c$ are constant such that

$$
\begin{aligned}
c \equiv 4 a^{2}+(n-2)\left[2 a \sum_{i=1}^{4} b_{i}\right. & +\left(\sum_{i=1}^{6}(-1)^{i-1} b_{i}\right)^{2} \\
& \left.-2\left(b_{1} b_{3}+b_{2} b_{4}-b_{5} b_{6}\right)+(n-1) \sum_{i=1}^{6} b_{i}^{2}\right]>0
\end{aligned}
$$

b's being any constants $\left({ }^{3}\right)$.

( $\left.{ }^{3}\right)$ An elementary calculation shows that $c \geqq 0$, where equality holds if and only if $b_{1}=\cdots=b_{4}, b_{5}=b_{6}=0, a=-(n-2) b_{1}$. 
For the case $a \neq 0, c-4 a^{2}=0$ and the case $a=0, c-4 a^{2} \neq 0$, Theorem $\mathrm{I}$ is due to Yano [10].

THEOREM II. A manifold $M^{n}$ is conformal to an $n$-sphere if it satisfies condition (C) and any one of the following three sets of conditions:

$$
\begin{aligned}
\nabla_{i} \nabla_{j}(R f) & =R \rho g_{i j} & & \text { ( } \text { is a scalar function }), \\
Q d \rho & =(2 / n) d(R \rho), & & \nabla_{i} \nabla_{j}(R \rho)=R \nabla_{i} \nabla_{j} \rho, \\
L_{v} R_{i j} & =\alpha g_{i j} & & (\alpha \text { is a scalar function }),
\end{aligned}
$$

where $Q$ is the operator of Ricci defined by, for any vector field $u$ on $M^{n}$,

$$
Q: u_{i} \rightarrow 2 R_{i j} u^{j} .
$$

For constant $R$, conditions (1.11) and (1.12) in Theorem II will lead to the conclusion that $M^{n}$ is isometric to an $n$-sphere of radius $(n(n-1) / R)^{1 / 2}$; for this see [12].

THEOREM III. A manifold $M^{n}$ with constant $R$ is isometric to an $n$-sphere of radius $(n(n-1) / R)^{1 / 2}$ if it satisfies conditions $(\mathrm{C})$ and $(1.10)$.

Theorem III is due to Lichnerowicz [6] when condition (1.10) is replaced by the following one:

$$
v \text { is the gradient of a scalar function } f \text {, i.e., } v_{i}=\nabla_{i} f \text {. }
$$

For constant $R$, it is easily seen that condition (1.14) is a special case of condition (1.10). In fact, in this case by using (1.1) condition (1.10) becomes $\nabla_{i} v_{j}+\nabla_{j} v_{i}$ $=2 \nabla_{i} \nabla_{j} f$, which is satisfied by $v_{i}=\nabla_{i} f+u_{i}$ where $u_{i}$ is any vector field generating an infinitesimal isometry.

THEOREM IV. A manifold $M^{n}$ is isometric to an $n$-sphere if it satisfies condition (C), $L_{d \rho} R=0$, and

$$
\begin{gathered}
A^{a} B^{b}=c=\text { const, } \\
c\left(\frac{2 a}{A}+\frac{(n-1) b}{B}\right)=\frac{2^{a}(a+b) R^{2(a+b-1)}}{n^{a+b-1}(n-1)^{a-1}},
\end{gathered}
$$

where $A, B$ are given by (1.8), and $a, b$ are nonnegative integers and not both zero.

For constant $R$, Theorem IV is due to Lichnerowicz [6] for $a=0, b=1$ and due to Hsiung [2] for general $a$ and $b$.

The following known theorems will be needed in the proofs of our Theorems I-IV.

Theorem A (YANo AND Nagano [11]). If a complete Einstein space $M^{n}$ of dimension $n>2$ admits an infinitesimal nonisometric conformal transformation, then $M^{n}$ is isometric to an $n$-sphere. 
TheOREM B (OBATA [7]). If a complete Riemannian manifold $M^{n}$ of dimension $n \geqq 2$ admits a nonconstant function $\rho$ such that $\nabla_{i} \nabla_{j} \rho=-c^{2} \rho g_{i j}$, where $c$ is a positive constant, then $M^{n}$ is isometric to an $n$-sphere of radius $1 / c$.

THEOREM C (TASHIRO [8]). If a complete Riemannian manifold $M^{n}$ of dimension $n>2$ admits a nonconstant function $\rho$ such that

$$
\nabla_{i} \nabla_{j} \rho=-(1 / n) g_{i j} \Delta \rho,
$$

then $M^{n}$ is conformal to an $n$-sphere.

THEOREM D (YANO [10]). An orientable manifold $M^{n}$ is conformal to an $n$-sphere if it satisfies condition $(\mathrm{C})$ and

$$
\left(R_{i j} \rho^{i} \rho^{j}-(1 / n(n-1)) R^{2} \rho^{2}, 1\right) \geqq 0 .
$$

2. Notation and formulas. In this section we shall list some known formulas (for the details of their derivations see Lichnerowicz' book [5, pp. 124-134] or Hsiung's paper [1]) which will be needed in the proofs to follow.

Let $v$ be a vector field defining an infinitesimal conformal transformation on a Riemannian manifold $M^{n}$ so that (1.1) holds. Then we have

$$
\begin{gathered}
\rho=-\delta v / n, \\
L_{v} R^{h}{ }_{i j k}=-\varepsilon_{k}^{h} \nabla_{i} \rho_{j}+\varepsilon_{j}^{h} \nabla_{i} \rho_{k}-g_{i j} \nabla_{k} \nabla^{h} \rho+g_{i k} \nabla_{j} \nabla^{h} \rho,
\end{gathered}
$$

where $\nabla^{h}=g^{i h} \nabla_{i}$, and $\varepsilon_{k}^{h}=1$ for $h=k$ and $=0$ for $h \neq k$. From (1.1) and (2.2) it follows immediately that

$$
\begin{gathered}
L_{v} R_{h i j k}=2 \rho R_{h i j k}-g_{h k} \nabla_{i} \rho_{j}+g_{h j} \nabla_{i} \rho_{k}-g_{i j} \nabla_{h} \rho_{k}+g_{i k} \nabla_{h} \rho_{j}, \\
L_{v} R_{i j}=g_{i j} \Delta \rho-(n-2) \nabla_{i} \rho_{j}, \\
L_{v} R=2(n-1) \Delta \rho-2 R \rho .
\end{gathered}
$$

For any scalar field $f$ and vector field $u$ on $M^{n}$, we have

$$
\begin{gathered}
\Delta f=-\nabla^{i} \nabla_{i} f, \\
(\Delta u)_{i}=-\nabla^{j} \nabla_{j} u_{i}+\frac{1}{2}(Q u)_{i},
\end{gathered}
$$

where $Q$ is the operator of Ricci defined by (1.13).

A necessary and sufficient condition for a vector field $v$ to define an infinitesimal conformal transformation on a compact manifold $M^{n}$ is that it satisfy

$$
\Delta v+(1-2 / n) d \delta v=Q v .
$$

For an infinitesimal transformation $v$ on a manifold $M^{n}$, we have

$$
\Delta \delta v=(1 /(n-1)) R \delta v-(n / 2(n-1)) L_{v} R .
$$

For any 1-form $\xi$ on a compact orientable manifold $M^{n}$ we have

$$
(\Delta \xi+(1-2 / n) d \delta \xi-Q \xi, \xi) \geqq 0,
$$

where the equality holds when and only when $\xi$ defines an infinitesimal conformal transformation on $M^{n}$. 
On the manifold $M^{n}$ consider the following tensors:

$$
\begin{gathered}
T_{i j}=R_{i j}-(1 / n) R g_{i j}, \\
T_{h i j k}=R_{h i j k}-(1 / n(n-1)) R\left(g_{i j} g_{h k}-g_{i k} g_{h j}\right), \\
W_{h i j k}=a T_{h i j k}+b_{1} g_{h k} T_{i j}-b_{2} g_{h j} T_{i k}+b_{3} g_{i j} T_{h k} \\
-b_{4} g_{i k} T_{h j}+b_{5} g_{h i} T_{j k}-b_{6} g_{j k} T_{h i},
\end{gathered}
$$

where $a$ and $b$ are constants. It is easily seen that

$$
g^{i j} T_{i j}=0, \quad g^{h k} T_{h i j k}=T_{i j} .
$$

Moreover, by (1.3), (1.5) and (2.13) we have

$$
\|W\|=a^{2} A+\frac{c-4 a^{2}}{n-2} B-\frac{1}{n}\left(\frac{2 a^{2}}{n-1}+\frac{c-4 a^{2}}{n-2}\right) R^{2},
$$

where $c$ is defined by (1.9).

3. Lemmas. Throughout this section $M^{n}$ will always denote a compact orientable Riemannian manifold of dimension $n \geqq 2$.

LEMMA 3.1. If $f$ is a scalar field on $M^{n}$ and $\Delta f=0$, then $f$ is constant.

Proof. From (2.6) and our assumption $\Delta f=0$, it follows that $\Delta\left(f^{2}\right)=-\nabla^{i} \nabla_{i}\left(f^{2}\right)$ $=-2\left(\nabla^{i} f\right)\left(\nabla_{i} f\right)$. By substituting $\nabla_{i}\left(f^{2}\right)$ for $\xi_{i}$ in the well-known Green's formula

$$
\int_{M^{n}} \nabla^{i} \xi_{i} d V=0
$$

where $\xi_{i}$ is any vector field on $M^{n}$, we therefore have

$$
0=\int_{M^{n}} \Delta\left(f^{2}\right) d V=-2 \int_{M^{n}}\left(\nabla^{i} f\right)\left(\nabla_{i} f\right) d V,
$$

which implies that $\nabla_{i} f=0$ since $\left(\nabla^{i} f\right)\left(\nabla_{i} f\right)$ is nonnegative.

LEMMA 3.2. For an orientable $M^{n}$ satisfying $L_{d \rho} R=0$ and (C) defined in $\S 1$, we have

$$
\left(R^{i j} \nabla_{i} \nabla_{j} \rho+R^{2} \rho / n(n-1), \rho\right) \geqq 0 .
$$

For constant $R$, Lemma 3.2 is due to Lichnerowicz [6].

Proof. By applying the integral formula (2.10) to the 1 -form $d \rho$ we have

$$
((2(n-1) / n) \Delta d \rho-Q d \rho, d \rho) \geqq 0 .
$$

On the other hand, covariant differentiation gives

$$
\begin{aligned}
& \nabla^{i}\left[\rho((2(n-1) / n) \Delta d \rho-Q d \rho)_{i}\right] \\
& \quad=\langle(2(n-1) / n) \Delta d \rho-Q d \rho, d \rho\rangle-\langle(2(n-1) / n) \Delta \Delta \rho-\delta Q d \rho, \rho\rangle .
\end{aligned}
$$


From (3.4), (3.5) and Green's formula (3.1) we thus obtain

$$
((2(n-1) / n) \Delta \Delta \rho-\delta Q d \rho, \rho) \geqq 0 .
$$

Due to the assumption $L_{v} R=0,(2.5)$ is reduced to

$$
\Delta \rho=R \rho /(n-1) \text {. }
$$

Since $L_{d \rho} R=0$ implies

$$
\rho^{i} \nabla_{i} R=0,
$$

substitution of $\rho^{2} \nabla_{i} R$ for $\xi_{i}$ in Green's formula (3.1) gives

$$
(\rho \Delta R, \rho)=0 .
$$

On the other hand, by the second Bianchi identity we have

$$
\nabla^{j} R_{i j}=\frac{1}{2} \nabla_{i} R
$$

which together with (3.8) implies

$$
\rho^{i} \nabla^{j} R_{i j}=0 .
$$

From (1.13), (3.11) and (3.7) follow immediately

$$
\begin{gathered}
\delta Q d \rho=-2 \nabla_{i}\left(R^{i j} \rho_{j}\right)=-2 R^{i j} \nabla_{i} \rho_{j}, \\
\Delta \Delta \rho=\left(1 /(n-1)^{2}\right) R^{2} \rho+(1 /(n-1)) \rho \Delta R .
\end{gathered}
$$

Substituting (3.12), (3.13) in (3.6) and making use of (3.9), we hence obtain the required inequality (3.3).

\section{Proofs of theorems.}

Proof of Theorem I. From (2.13) and the condition $L_{v} R=0$ it follows that

$$
L_{v}\|W\|=L_{v}\left(a^{2} A+\frac{c-4 a^{2}}{n-2} B\right)
$$

By means of (2.13), (2.12), (1.1), (2.3), (2.4), (3.7) we can easily compute $L_{v} W_{h i j k}$ (for the details see [3, p. 189]), and then multiplying both sides of the resulting expression by $W^{i j k}$ and making use of (2.13), (2.12), (1.1), (2.15), (1.9) and $R_{i j k}^{i}=0$ an elementary but lengthy calculation yields

$$
W^{h i j k} L_{v} W_{h i j k}=2 \rho\|W\|-c T^{i j} \nabla_{i} \rho_{j} .
$$

Substitution of (4.2) in the well-known formula

$$
L_{v}\|W\|=2 W^{h i j k} L_{v} W_{h i j k}-8 \rho\|W\|
$$

thus gives

$$
\rho L_{v}\|W\|=-4 \rho^{2}\|W\|-2 c \rho T^{i j} \nabla_{i} \rho_{j} .
$$


A straightforward computation and use of (2.6), (3.7), (2.11), (3.10) can easily show that

$$
\begin{gathered}
\nabla^{i}\left(R \rho \rho_{i}\right)=\left(\nabla_{i} R\right) \rho \rho^{i}+R \rho_{i} \rho^{i}-R^{2} \rho^{2} /(n-1), \\
\nabla^{i}\left(T_{i j} \rho \rho^{j}\right)=R_{i j} \rho^{i} \rho^{j}+\rho T^{i j} \nabla_{i} \rho_{j}+((n-2) / 2 n)\left(\nabla_{i} R\right) \rho \rho^{i}-(1 / n) R \rho_{i} \rho^{i} .
\end{gathered}
$$

By substituting (4.5) for $\left(\nabla_{i} R\right) \rho \rho^{i}$ and (4.4) for $\rho T^{i j} \nabla_{i} \rho_{j}$ in (4.6), integrating over $M^{n}$ and making use of (3.1), we thus obtain

$$
\begin{aligned}
& 2 c\left(R_{i j} \rho^{i} \rho^{j}-(1 / n(n-1)) R^{2} \rho^{2}, 1\right) \\
& \quad=4\left(\|W\|, \rho^{2}\right)+\left(L_{v}\|W\|, \rho\right)+c\left(\rho_{i} \rho^{i}-(1 /(n-1)) R \rho^{2}, R\right) .
\end{aligned}
$$

Since $\left(\|W\|, \rho^{2}\right)$ is nonnegative, from (4.7), (4.1) and our assumption (1.6), (1.7) we obtain (1.18). Hence by Theorem $\mathrm{D}, M^{n}$ is conformal to an $n$-sphere.

Proof of Theorem II. First suppose (1.10) holds. Then from (1.10), (2.6) it follows that $\Delta(R f)=-n R \rho$, implying, together with (3.7), that

$$
\Delta(\rho+(1 / n(n-1)) R f)=0 .
$$

Thus by Lemma 3.1, $\rho+(1 / n(n-1)) R f$ is a constant. Using (1.10), (3.7) we therefore obtain

$$
\nabla_{i} \rho_{j}=-(1 / n(n-1)) \nabla_{i} \nabla_{j}(R f)=-(1 / n) g_{i j} \Delta \rho .
$$

Hence by Theorem C, $M^{n}$ is conformal to an $n$-sphere.

Next suppose (1.11) holds. From the definition of $\Delta$ it follows that

$$
d \Delta \rho=\Delta d \rho,
$$

which, together with (3.7) and the first equation of (1.11), implies

$$
\Delta d \rho+(1-2 / n) d \delta d \rho-Q d \rho=0 .
$$

Thus by the necessary and sufficient condition (2.8) we see that $d \rho$ generates an infinitesimal conformal transformation on $M^{n}$ so that $L_{d \rho} g_{i j}=2 \phi g_{i j}$, which shows

$$
\nabla_{i} \rho_{j}=\phi g_{i j},
$$

where $\phi \neq 0$ in consequence of (3.7). From (4.11) and the second equation of (1.11) it follows that

$$
\nabla_{i} \nabla_{j}(R \rho)=R \phi g_{i j} .
$$

Thus the condition (1.10) is satisfied for $v=d \rho$ and $f=\rho$, and hence $M^{n}$ is conformal to an $n$-sphere.

Finally suppose (1.12) holds. Then (2.4) becomes

$$
\alpha g_{i j}=g_{i j} \Delta \rho-(n-2) \nabla_{i} \rho_{j} .
$$

Multiplying (4.13) by $g^{i j}$ and using (3.7) we obtain

$$
\alpha=(2 / n) R \rho .
$$


Substitution of (4.14), (3.7) in (4.13) thus gives (1.17), and hence Theorem C completes the proof of our theorem.

Proof of Theorem III. It is exactly the same as that of Theorem II for condition (1.10) except that the application of Theorem $\mathrm{C}$ should be replaced by that of Theorem B.

Proof of Theorem IV. Without loss of generality we may assume our manifold $M^{n}$ to be orientable as otherwise we need only to take an orientable twofold covering space of $M^{n}$. On the manifold $M^{n}$ consider the covariant tensor field $T$ of order $2(2 a+b)$ :

$$
\begin{aligned}
& T_{h_{1} i_{1} j_{1} k_{1} \cdots h_{a} i_{a} j_{a} k_{a} u_{1} v_{1} \cdots u_{b} v_{b}} \\
& \quad=\prod_{r=1}^{a} R_{h_{r} i_{r} j_{r} k_{r}} \prod_{s=1}^{b} R_{u_{s} v_{s}}-\frac{R^{a+b}}{n^{a+b}(n-1)^{a}} \prod_{r=1}^{a}\left(g_{i_{r} j_{r}} g_{h_{r} k_{r}}-g_{i_{r} k_{r}} g_{h_{r} j_{r}}\right) \prod_{s=1}^{b} g_{u_{s} v_{s} .} .
\end{aligned}
$$

From (4.15) an elementary calculation gives the length of $T$ :

$$
[2(2 a+b)] !\langle T, T\rangle=A^{a} B^{b}-2^{a} R^{2(a+b)} / n^{a+b}(n-1)^{a} .
$$

Thus by condition (1.15), $L_{v} R=0$ and the extension of formula (4.3) to the tensor $T$ we immediately obtain

$$
L_{v}\langle T, T\rangle=2\left\langle L_{v} T, T\right\rangle-4(2 a+b) \rho\langle T, T\rangle=0,
$$

which implies

$$
\left(\left\langle L_{v} T, T\right\rangle, \rho\right)=2(2 a+b)(\rho\langle T, T\rangle, \rho) .
$$

On the other hand, from (2.3), (2.4) we obtain

$L_{v} T_{h_{1} i_{1} j_{1} k_{1} \cdots h_{a} i_{a} j_{a} k_{a} u_{1} v_{1} \cdots u_{b} v_{b}}$

$$
\begin{aligned}
& =2 a \rho \prod_{r=1}^{a} R_{h_{r} i_{r} j_{r} k_{r}} \prod_{s=1}^{b} R_{u_{s} v_{s}} \\
& -\sum_{r=1}^{a}\left[R_{h_{1} i_{1} j_{1} k_{1}} \cdots R_{h_{r-1} i_{r-1} j_{r-1} k_{r-1}}\right. \\
& \cdot\left(g_{h_{r} k_{r}} \nabla_{i_{r}} \nabla_{j_{r}} \rho-g_{h_{r} j_{r}} \nabla_{i_{r}} \nabla_{k_{r}} \rho+g_{i_{r} j_{r}} \nabla_{k_{r}} \nabla_{h_{r}} \rho-g_{i_{r} k_{r}} \nabla_{j_{r}} \nabla_{h_{r}} \rho\right) \\
& \left.\cdot R_{h_{r+1} i_{r+1} j_{r+1} k_{r+1}} \cdots R_{h_{a} i_{a} j_{a} k_{a}}\right] \prod_{s=1}^{b} R_{u_{s} v_{s}} \\
& +\prod_{r=1}^{a} R_{h_{r} i_{r} j_{r} k_{r}} \sum_{s=1}^{b}\left\{R_{u_{1} v_{1}} \cdots R_{u_{s-1} v_{s-1}}\right. \\
& \left.\cdot\left[g_{u_{s} v_{s}} \Delta \rho-(n-2) \nabla_{v_{s}} \nabla_{u_{s}} \rho\right] \cdot R_{u_{s}+1 v_{s}+1} \cdots R_{u_{b} v_{b}}\right\} \\
& -\frac{2(2 a+b) R^{a+b}}{n^{a+b}(n-1)^{a}} \rho \prod_{r=1}^{a}\left(g_{i_{r} j_{r}} g_{h_{r} k_{r}}-g_{i_{r} k_{r}} g_{h_{r} j_{r}}\right) \prod_{s=1}^{b} g_{u_{s} v_{s}} \text {. }
\end{aligned}
$$

By means of (4.15), (4.19), (3.7), (1.16), (1.8), (4.16) an elementary calculation yields

$$
\begin{aligned}
\left(\left\langle L_{v} T, T\right\rangle, \rho\right)=2 a & (\rho\langle T, T\rangle, \rho) \\
& -\frac{A^{a} B^{b}}{[2(2 a+b)] !}\left(\frac{4 a}{A}+\frac{(n-2) b}{B}\right)\left(R^{j k} \nabla_{j} \nabla_{k} \rho+\frac{R^{2} \rho}{n(n-1)}, \rho\right) .
\end{aligned}
$$


By comparing (4.18) and (4.20), noticing that $\rho \neq 0$, and making use of Lemma 3.2, we thus have

$$
T_{h_{1} i_{1} j_{1} k_{1} \cdots h_{a} i_{a} j_{a} k_{a} u_{1} v_{1} \cdots u_{b} v_{b}}=0 .
$$

Multiplying (4.21) by

$$
g^{h_{1} k_{1}} \prod_{r=2}^{a} g^{h_{r} k_{r}} g^{i_{r} j_{r}} \prod_{s=1}^{b} g^{u_{s} v_{s}}
$$

and using (4.15) we obtain $R_{i_{1} j_{1}}=R g_{i_{1} j_{1}} / n$, which implies $M^{n}$ is an Einstein space. Hence, by Theorem A, $M^{n}$ is isometric to an $n$-sphere, and our theorem is proved.

\section{BIBLIOGRAPHY}

1. C. C. Hsiung, Vector fields and infinitesimal transformations on Riemannian manifolds with boundary, Bull. Soc. Math. France 92 (1964), 411-434. MR 31 \#2693.

2. - On the group of conformal transformations of a compact Riemannian manifold, Proc. Nat. Acad. Sci. U.S.A. 54 (1965), 1509-1513. MR 32 \#6372.

3. - On the group of conformal transformations of a compact Riemannian manifold. III, J. Differential Geometry 2 (1968), 185-190. MR 38 \#1637.

4. C. C. Hsiung and L. R. Mugridge, Conformal changes of metrics on a Riemannian manifold, Math. Z. 119 (1971), 179-187.

5. A. Lichnerowicz, Géométrie des groupes de transformations, Dunod, Paris, 1958.

6. - Sur les transformations conformes d'une variété riemannienne compacte, C. R. Acad. Sci. Paris 259 (1964), 697-700. MR 29 \#4007.

7. M. Obata, Certain conditions for a Riemannian manifold to be isometric with a sphere, J. Math. Soc. Japan 14 (1962), 333-340. MR 25 \#5479.

8. Y. Tashiro, Complete Riemannian manifolds and some vector fields, Trans. Amer. Math. Soc. 117 (1965), 251-275. MR 30 \#4229.

9. K. Yano, On harmonic and Killing vector fields, Ann. of Math. (2) 55 (1952), 38-45. MR 13, 689.

10. - On Riemannian manifolds admitting an infinitesimal conformal transformation Math. Z. 113 (1970), 205-214. MR 41 \#6114.

11. K. Yano and T. Nagano, Einstein spaces admitting a one-parameter group of conformal transformations, Ann. of Math. (2) 69 (1959), 451-461. MR 21 \#345.

12. K. Yano and M. Obata, Sur le groupe de transformations conformes d'une variété de Riemann dont le scalaire de courbure est constant, C. R. Acad. Sci. Paris 260 (1965), 2698-2700. MR 31 \#697.

13. - Conformal changes of Riemannian metrics, J. Differential Geometry 4 (1970), 53-72. MR 41 \#6113.

Lehigh University, Bethlehem, Pennsylvania 18015 